\title{
PLANAR FOURIER TRANSFORMS AND DIOPHANTINE APPROXIMATION
}

\author{
R. KAUFMAN ${ }^{1}$
}

\begin{abstract}
The radial behavior of its Fourier-Stieltjes transform in $R^{2}$ is related to the modulus of continuity of a measure; in certain cases the Hausdorff dimension of an exceptional set of lines can be estimated. Converse results use the theory of Diophantine approximation established by Busicovitch and Jarník.
\end{abstract}

1. We shall study metrical properties of a measure $\mu \geqq 0$ of compact support in the plane $R^{2}$, relating these properties to the behavior of the Fourier-Stieltjes transform $\hat{\mu}$ on lines through $(0,0)$. The simplest of the metrical properties is the Lipschitz condition of order $\alpha: \mu \in \Lambda_{\alpha}$ shall mean that $\mu(S) \ll(\operatorname{diam} S)^{x}$ for all planar sets $S$. A set of lines through $(0,0)$ is said to have a property $\mathrm{P}$, if that property is valid for the corresponding set of directions $\theta$ in $[0,2 \pi)$.

THEOREM 1. If $\mu \in \Lambda_{\alpha}, 1<\alpha<2$, then $\hat{\mu}$ tends to 0 along all lines, except a set of Hausdorff dimension at most $2-\alpha$. The upper bound $2-\alpha$ cannot be improved.

THEOREM 2. The transform $\hat{\mu}$ tends to 0 along almost all lines, provided $\iint|x-y|^{-1} \mu(d x) \mu(d y)<\infty$.

Theorem 4 is a converse to this.

2. It is convenient to write transforms as $\hat{\mu}(r \xi)$, with $r \geqq 0$ and $|\xi|=1$, and to write $d \xi$ for normalized arc-length on the circle $|\xi|=1$. The Bessel function of order 0 is

$$
J_{0}(u) \equiv \int \exp i(x, \xi) d \xi, \quad \text { if }|x|=u \geqq 0 .
$$

Concerning $J_{0}$ we need to know that it has successive integrals $\varphi_{0}=J_{0}, \varphi_{1}$, and $\varphi_{2}$ each satisfying $|\varphi(u)| \ll(1+|u|)^{-1 / 2}$ on the real axis. This follows

Received by the editors August 21, 1972 and, in revised form, October 17, 1972.

AMS (MOS) subject classifications (1970). Primary 42A72, 10K15; Secondary $28 \mathrm{~A} 75$.

Key words and phrases. Fourier-Stieltjes transform, Hausdorff dimension, $J_{0}$, Diophantine approximation.

${ }^{1}$ Alfred E. Sloan Fellow. 
from the asymptotic expansion for $J_{0}$ up to $O\left(u^{-4}\right)$; see [4, pp. 46-51] or $[3, \mathrm{XIV}-\mathrm{XV}]$.

The following identity is basic, and is stated for a complex measure $\mu$; $f(r)$ is a suitable function of $r$.

$$
\begin{aligned}
\iint f(r)|\hat{\mu}(r \xi)|^{2} d r d \xi & =\int \cdots \int \exp \operatorname{ir}(\xi, x-y) \mu(d x) \overline{\mu(d y)} d r d \xi \\
& =\iiint f(r) J_{0}(r|x-y|) \mu(d x) \overline{\mu(d y)} d r .
\end{aligned}
$$

The problems at hand require bounds in which $f(r)$ is the characteristic function of an interval, but $\int_{R}^{2 R} J_{0}(r X) d r$ does not decrease rapidly enough to obtain the necessary bounds, when $\alpha \geqq 3 / 2$. The following device is borrowed from summability: let $F \in C^{2}(-\infty, \infty)$ vanish outside $(1 / 2$, $5 / 2$ ), let $F \geqq 1$ on $[1,2]$ and $F \geqq 0$ everywhere.

Lemma 1. $\left|\int F\left(R^{-1} r\right) J_{0}(r X) d r\right| \ll \min \left(R, R^{-3 / 2} X^{-5 / 2}\right)$ for all $R>0, X>0$.

In fact, we can integrate by parts to obtain

$$
R^{-2} X^{-2}\left|\int F^{\prime \prime}\left(R^{-1} r\right) \varphi_{2}(r X)\right| \ll R^{-2} X^{-2} \int_{R_{2}}^{3 R}|r X|^{-1 / 2} d r .
$$

This yields the bound $R^{-3 / 2} X^{-5 / 2}$, and the bound $\ll R$ follows from $\left|J_{0}\right| \leqq 1$.

To prove the first part of Theorem 1 we observe that

$$
\begin{aligned}
\int_{R}^{2 R} \int|\hat{\mu}(r \xi)|^{2} d r d \xi & \leqq \iint F\left(R^{-1} r\right)|\hat{\mu}(r \xi)|^{2} d r d \xi \\
& =\iiint \int F\left(R^{-1} r\right) J_{0}(r|x-y|) d r \mu(d x) \overline{\mu(d y)} \\
& \ll \iint \min \left(R, R^{-3 / 2}|x-y|^{-5 / 2}\right) \mu(d x) \mu(d y) .
\end{aligned}
$$

Let $P(t)$ be the product measure of the set $\{|x-y| \leqq t\}$, so that $P(t) \ll t^{\alpha}$ when $\mu \in \Lambda_{\alpha}$. The last integral is then

$$
\begin{aligned}
\int \min \left(R, R^{-3 / 2} t^{-5 / 2}\right) d P(t) & \leqq R P\left(R^{-1}\right)+3 R^{-3 / 2} \int_{R^{-1}}^{\infty} t^{-7 / 2} P(t) d t \\
& \ll R^{1-\alpha}, \text { as } \alpha \leqq 2 .
\end{aligned}
$$

Now suppose $R>e$, and there is an $r$ in $[R, 2 R]$ such that $\left.|\hat{\mu}(r \xi)|^{2}\right\rangle$ $(\log R)^{-1}$. Since $\hat{\mu}$ has bounded partial derivatives in the plane, we have $\int_{R}^{2 R}|\hat{\mu}(r \xi)|^{2} d r>c(\log R)^{-2}$, and in fact the same inequality holds on an arc about $\xi$ of length $c(\log R)^{-1} R^{-1}$. Dividing the circumference $|\xi|=1$ into arcs $I$ of length approximately $R^{-1}$, and using the estimate for 
$\int_{R}^{2 R} \int(\cdot) d r d \xi$ obtained above, we see that the number $N_{R}$ of $\operatorname{arcs} I$ such that $\sup \left\{|\hat{\mu}(r \xi)|^{2}: R \leqq r \leqq 2 R\right\}>(\log R)^{-1}$ somewhere in $I$, has order of magnitude $N_{R} \ll R^{2-x}(\log R)^{3}$. Letting $R=1,2, \cdots, 2^{k}, \cdots$, we see that $\hat{\mu}(r \xi) \ll(\log r)^{-1}$ except on a $\xi$-set of Hausdorff dimension $\leqq 2-\alpha$; this is the first assertion in Theorem 1.

To prove Theorem 2 we prove in fact that $\hat{\mu}$ is in $L^{2}$ along almost all lines. Indeed

$$
\int_{0}^{R} \int|\hat{\mu}(r \xi)|^{2} d r d \xi=\int_{0}^{R} \iint J_{0}(r|x-y|) \mu(d x) \mu(d y) d r .
$$

But $J_{0}$ has a bounded primitive, whence

$$
\int_{0}^{R} \int|\hat{\mu}(r \xi)|^{2} d r d \xi \leqq C \iint|x-y|^{-1} \mu(d x) \mu(d y)<\infty .
$$

4. We show that the set of exceptional directions can attain the dimension $2-\alpha$; when $\alpha=1$ we obtain a precise converse to Theorem 2 . The symbol $\|u\|$ now denotes the distance between a real number $u$ and the nearest integer; plane vectors are denoted $X, Y$ and lowercase letters denote real numbers.

Henceforth $\left(N_{k}\right)_{1}^{\infty}$ is always a strictly increasing sequence of positive integers and $\varepsilon(k)>0$ a function of natural numbers, tending to 0 . Given $\eta$ in $(0,1]$ the set $F=F(N, \varepsilon, \eta)$ is defined

$$
F:-1 \leqq x \leqq 1, \quad\left\|N_{k} x\right\| \leqq \varepsilon(k) N_{k}^{-\eta}, \quad 1 \leqq k<\infty .
$$

For an appropriate sequence $N, \operatorname{dim} F \geqq(1+\eta)^{-1}$. For example $F$ can be a dyadic set, the set of all sums $\sum \pm 2^{-m_{j}}$ where $1 \leqq m_{1}<\cdots<m_{j}<$ $m_{j+1}<\cdots$ and $m_{j} \leqq(1+\eta) j+o(j)$. We can set $N_{k}=2^{m_{j}}$ provided $m_{j+1} \geqq$ $(1+\eta) m_{j}+1-\log \varepsilon(k) / \log 2$; the existence of a sequence of 'exceptional' indices $m_{j+1}$ is compatible with the first condition. Also, $F$ is represented as a product set and thus carries a product measure, which is in $\Lambda_{c}$ for all $c<(1+\eta)^{-1}$. (The Lipschitz condition can be established from elementary properties of binary expansions.) Hence $F \times F$ carries a product measure in each class $\Lambda_{2 c}, c<(1+\eta)^{-1}$.

Let $H$ be the set of real numbers $t$ for which the Diophantine inequalities

$$
1 \leqq v \leqq \varepsilon^{-1 / 2}(k) N_{r}^{\eta}, \quad\|v t\| \leqq \varepsilon^{1 / 3}(k) N_{k}^{-1}
$$

have an integer solution for each $k=1,2, \cdots$. For this integer $v$, and $x$ in $F$,

$$
\left\|v N_{k} x\right\| \leqq v\left\|N_{k} x\right\| \leqq \varepsilon^{1 / 2}(k), \quad\left\|v N_{k} t x\right\| \leqq\|v t u\|+|v t|\left|N_{k} x-u\right|
$$


for any integer $u$. Choosing $u=u(x)$ to be the integer closest to $N_{k} x$ we find

whence

$$
|u| \leqq 1+N_{k}, \quad\left|N_{k} x-u\right| \leqq \varepsilon(k) N_{k}^{-\eta},
$$

$$
\left\|v N_{k} t x\right\| \leqq 2 N_{k} \cdot \varepsilon^{1 / 3}(k) N_{k}^{-1}+|t| \varepsilon^{1 / 2}(k)=o(1) .
$$

By the method of Jarniik ([1], [2], [5], [6]), when $\left(N_{k}\right)$ is sufficiently sparse, $\operatorname{dim} H=2\left(1+\eta^{-1}\right)^{-1}=2 \eta(1+\eta)^{-1}$. In order to apply the arguments cited, it is easier in fact to treat a smaller set, defined by stronger inequalities

$$
\frac{1}{2} N_{k}^{\eta} \leqq v \leqq N_{k}^{\eta}, \quad\|v t\| \leqq \varepsilon^{1 / 3}(k) N_{k}^{-1} .
$$

(Jarník's method can be simplified somewhat by the use of Borel measures [7].) Setting $\alpha=2(1+\eta)^{-1}, 2-\alpha=2 \eta(1+\eta)^{-1}$ we can state

THEOREM 3. There exists a probability measure $\sigma$ on the line, in each class $\Lambda_{c}, c<2 \alpha$, and a set $H$ of dimension $2-\alpha$, such that for each $t$ in $H$, there is a sequence $r_{k}(t) \rightarrow \infty$ with $\lim \left\|r_{k} x\right\|=\lim \left\|r_{k} t x\right\|=0$, uniformly for all $x$ in the closed support of $\sigma$.

Theorem 3 complements Theorem 2 and establishes the exponent $2-\alpha$ as best-possible; for $\mu=\sigma \times \sigma$ belongs to $\Lambda_{c}$ whenever $c<\alpha$, but $\hat{\mu}\left(2 \pi r_{k}(t), 2 \pi r_{k}(t) t\right) \rightarrow 1$ so that the exceptional set of directions includes $H$, and hence has dimension exactly $2-\alpha$.

The calculations in Theorem 3 can be applied when $\eta=1$; here Dirichlet's principle yields a solution of (II) for every real $t$, so that the transform $\hat{\mu}$ tends to zero along $n o$ line (the vertical line corresponding to $t=\infty$ is easily handled separately). Thus, to investigate the rôle of the integral in Theorem 2, we construct sets $F(N, \varepsilon, 1)$ as massive as possible.

THEOREM 4. Let $f(r)$ be a decreasing positive function on $(0<r<\infty)$, and $f(0+)=+\infty$. Then for an appropriate sequence $N=\left(N_{k}\right)$, and $\varepsilon(k) \rightarrow 0$, the set $F=F(N, \varepsilon, 1)$ carries a measure $\sigma$ whose modulus of continuity $\omega$, defined by

has the property

$$
\omega(y)=\sup \sigma(B), \quad \operatorname{diam} B \leqq y,
$$

$$
\int_{r}^{\infty} \omega^{2}(y) y^{-2} d y \ll f(r), \quad 0<r<1 .
$$

When $\mu=\sigma \times \sigma$, in the plane, we have for any vector $Y$,

$$
\int_{|X|-Y \geqq r}|X-Y|^{-1} \mu(d X) \ll \int_{r}^{\infty} \omega^{2}(y) y^{-2} d y,
$$

so this theorem completes Theorem 2 . 
The canonical product measure on the dyadic set $F_{0}$ of all sums $\sum \pm 2^{-m_{j}}$ has modulus of continuity $\omega\left(2^{-m_{j}}\right) \ll 2^{-j}$. Moreover, $F_{0}$ will be contained in a set $F(N, \varepsilon, 1)$ provided $\sup \left(m_{j+1}-2 m_{j}\right)=+\infty$. To estimate the integral $\int_{r}^{\infty}$, we suppose that $r=2^{-m_{s}}$ and sum over the intervals $2^{-m_{2}} \leqq y \leqq$ $2^{-m_{1}}, \cdots$, obtaining

$$
\int_{r}^{\infty} \omega^{2}(y) y^{-2} d y \ll \sum_{1}^{s} 4^{-m_{j}} 2^{m_{j}} \equiv \Phi(s), \quad \text { when } r=2^{-m_{\varepsilon}} .
$$

Suppose now that $M=\left(m_{j}\right)$ is an increasing sequence of positive integers, that $m_{j+1}=m_{j}+1$ for $j \geqq j_{1}$ and $\Phi(s) \leqq c f\left(s^{-1}\right)$ for $s \geqq 1$. We claim that if $p$ is sufficiently large the sequence $\bar{M}$ defined by

$$
\bar{m}_{j}=m_{j}, \quad 1 \leqq j<p, \quad \bar{m}_{j}=m_{j}+m_{p}+k, \quad p \leqq j<\infty
$$

has the same properties. In fact $\Phi(s)$ is unchanged when $s<p$, and otherwise $\Phi(s)$ is increased by

$$
2^{m_{p}} 2^{k} \sum_{p}^{s} 4^{-j} 2^{m_{j}} \leqq 2^{k+1} 4^{m_{p}} 4^{-p}
$$

Using again the relation $m_{j+1}=m_{j}+1$ for $j \geqq j_{1}$ we can estimate this increment by $B_{k}$, so the inequality on $p$ becomes

$$
\Phi(s)+B_{k} \leqq c f\left(s^{-1}\right) \quad \text { for } s \geqq p .
$$

Now $f(0+)=+\infty$ while $\Phi(s)$ remains bounded, so the inequality written above is satisfied for large $p$. In the sequence $\bar{M}$ we have $\bar{m}_{p} \geqq 2 \bar{m}_{p-1}+k$. This process, repeated for $k=1,2,3, \cdots$, yields a sequence with the necessary gaps, while $\Phi(s) \leqq c f\left(s^{-1}\right)$ for $s \geqq 1$, and hence $4^{-s} 2^{m_{s}} \leqq c f\left(s^{-1}\right)$. Thus if $f(t)<t^{-1}$ near 0 we have $m_{s}<3 s$ for large $s$. To each small $r>0$ there is an $s$ such that $r^{4} \leqq 2^{-m_{s}}<r$, and

$$
\int_{r}^{\infty} \omega^{2}(y) y^{-2} d y \ll \Phi(s) \leqq c f\left(s^{-1}\right) \leqq c f(r) .
$$

Although we have a satisfactory converse to Theorem 2, it remains to construct a set $E$ of positive 1-measure or, if possible, non- $\sigma$-finite 1measure, such that $E$ carries no measure $\mu$ whose Fourier-Stieltjes transform $\hat{\mu}(r \cos \theta, r \sin \theta)$ tends to 0 along almost all lines. Unfortunately all the sets $F(N, \varepsilon, 1)$ have 1-measure 0 .

\section{REFERENCES}

1. A. Baker and W. Schmidt, Diophantine approximation and Hausdorff dimension, Proc. London Math. Soc. (3) 21 (1970), 1-11. MR 42 \#5916.

2. A. S. Besicovitch, Sets of fractional dimensions. IV: On rational approximations to real numbers, J. London Math. Soc. 9 (1934), 126-131. 
3. J. Dieudonné, Calcul infinitesimal, Hermann, Paris, 1968. MR 37 \#2557.

4. A. Erdélyi, Asymptotic expansions, Dover, New York, 1956. MR 17, 1202.

5. V. Jarník, Über einen Satz von A. Khintchine, Acta Arith. 2 (1937), 1-22.

6. - Über die simultanen diophantischen Approximationen, Math. Z. 33 (1931), $505-543$.

7. R. Kaufman, Probability, Hausdorff dimension, and fractional distribution, Mathematika 17 (1970), 63-67. MR 42 \#7619.

Department of Mathematics, University of Illinois, Urbana, Illinois 61801 